\title{
Improved Artificial Bee Colony Algorithm Applied to Neural Network Photovoltaic Power Generation Prediction Method
}

\author{
Jiaxiong $\mathrm{ZHU}^{1}$, JIANG QIANG ${ }^{2}$, CHANG FENG ${ }^{3}$ and $\mathrm{CAO} \mathrm{JING}^{1 *}$ \\ ${ }^{1}$ School of Physics And Electronic Engineering of Leshan Normal university, Leshan 614004, China \\ ${ }^{2}$ School of Physics And Electronic Engineering of Leshan Normal university, Leshan \\ 614004, China \\ ${ }^{3}$ School of Physics And Electronic Engineering of Leshan Normal university, Leshan \\ 614004, China
}

\begin{abstract}
With the increase in the use of renewable energy, especially the development and utilization of solar energy resources, accurate photovoltaic power generation prediction technology will help the promotion of photovoltaic power generation. The amount of photovoltaic power generation depends on weather conditions, and it is easy to produce large fluctuations under different weather conditions. Its power generation has the characteristics of randomness, fluctuation and intermittency. In view of the shortcomings of the traditional BP neural network prediction method, this paper proposes an improved artificial bee colony algorithm. The improved artificial bee colony algorithm is used to optimize the network parameter weights in the traditional BP algorithm, and the two algorithms are merged in global iteration. Based on the characteristics of training light intensity, weather, temperature and historical power value of photovoltaic output power,a photovoltaic power generation prediction model is established. The simulation results show that the improved artificial bee colony algorithm in the neural network's photovoltaic power generation forecast improves the accuracy and convergence speed of the traditional BP neural network convergence solution, and can provide more comprehensive information for grid power dispatch and control.
\end{abstract}

\section{Introduction}

The stability of photovoltaic power generation is poor, affected by a variety of meteorological factors, with significant intermittency and uncertainty. When photovoltaic power generation has a random impact on the power grid, it affects the dispatch and planning of the power grid and the safe operation of the power system. To solve this problem, the power grid generally provides backup units for the photovoltaic grid-connected power generation system to combat its output power fluctuations, and to ensure the safe operation of the power grid at the expense of economy. Therefore, the photovoltaic power generation forecast is an important basis for the dispatch department to adjust the dispatch plan and distribute the load reasonably, and it is also an important technical measure to reduce the impact of the photovoltaic system access on the power grid. There are many factors influencing photovoltaic power generation. The accurate prediction of photovoltaic power generation is complicated. The traditional $\mathrm{BP}^{[1]}$ neural network prediction method has large errors and slow convergence speed. It is also sensitive to the selection of initial values, making it difficult to predict accurately.

Literature $^{[2]}$ established an improved BP neural network photovoltaic power short-term prediction model, using a combination of increased momentum terms and variable learning rate to improve the neural network, in order to improve prediction accuracy, lead to similar day selection and training sample determination To directly predict the output power.

Reference ${ }^{[3]}$ based on the principle of similar days and BP neural network prediction method, using historical weather information of photovoltaic power plants, based on Manhattan distance to find similar days, directly predict photovoltaic power generation.

Reference ${ }^{[4]}$ introduces genetic algorithm on the basis of BP neural network, and improves the selection of initial connection weights and thresholds of BP neural network to achieve precise location of distribution network faults.

Reference ${ }^{[5]}$ uses artificial neural network method to predict the load model parameters of the next day's maximum and minimum load time, and then takes the minimum load active model prediction as an example to make the actual prediction.

This paper proposes an improved artificial bee colony algorithm in the neural network photovoltaic generation prediction method, improves the artificial bee colony algorithm, further strengthens its search speed and optimization ability, and then applies this improved

*Corresponding author's e-mail: 29265599@qq.com 
artificial bee colony algorithm to BP neural network. On the network, a new hybrid neural network is obtained, and then the time correlation between similar daily power generation data under different light intensity, temperature, and weather conditions is fully utilized to find the optimal solution and improve the convergence speed and accuracy of the neural network.

\section{Artificial Bee Colony Algorithm}

In the $\mathrm{ABC}$ algorithm ${ }^{[5-6]}$, the entire bee colony is divided into three parts: collecting bees, following bees and detecting bees, and introducing the concepts of honey source and fitness. What honey source represents is the possible solution to the problem. The bee colony first generates $\mathrm{N}$ honey sources to enter the nectar collection stage. The bee collector searches in the neighborhood of each honey source and generates a new honey source. By calculating the fitness of the honey source, it compares the pros and cons of the old and new honey sources and retains the better quality honey sources. The bee-collecting bee passes the fitness of the searched honey source to the following bee. In the following phase, the following bees select the honey source with a certain probability according to the adaptability of the honey source, and the honey source with high return rate has a high probability of being selected. After selecting the honey source, the following bees also search and generate a new honey source in the neighborhood of the honey source, compare the pros and cons of the two honey sources, and retain the good quality honey source. The bee colony seeks the optimal solution of the problem through the cycle of finding the honey source. When the individual goes through several generations of cycles continuously and the honey source is not updated, then the honey source is abandoned to become a detective bee, looking for a new honey source.

The formula for searching new honey sources in the $\mathrm{ABC}$ algorithm for collecting bees and following bees is:

$$
X_{x j}^{n e w}=X_{i j}+r \times\left(X_{x j}-X_{n e i g h b o u r, j}\right)
$$

Among them, $r$ is a random number of $(0,1)$, neighbour is a natural number and $n e i g h b o u r \neq i, j \in\{1,2, \cdots, m\} \quad$ randomly selected values.

The probability value $P_{i}$ corresponding to the bee colony and solution $X_{i}$ is:

$$
P_{i}=\frac{f i t_{i}}{\sum_{j=1}^{m} f i t_{j}}
$$

Among them, fit ${ }_{i}$ is the fitness corresponding to $X_{i}$.

\section{Improved Artificial Bee Colony Algorithm}

In order to improve the optimization accuracy and convergence speed of the ABC algorithm, this paper improves the algorithm in both the search method and the following bee selection probability.

\subsection{Improve Search Methods}

In order to speed up the convergence rate, a global optimal solution is introduced when searching, the search formula is:

$$
X_{x j}^{n e w}=X_{i j}+r_{1} \times\left(X_{x j}-X_{n e i g h b o u r, j}\right)+r_{2}\left(y_{j}-x_{x j}\right)
$$

Among them, $r_{1}$ is a random number of $(0,1), r_{2}$ is a random number of $(0,1.5)$, and $y_{j}$ is the jth variable of the global optimal solution.

After introducing the global optimal solution, the $\mathrm{ABC}$ algorithm will concentrate on the honey source with high adaptability and destroy the diversity of the bee colony, so that the premature convergence phenomenon occurs and falls into the local minimum. In order to balance the convergence speed and the diversity of the population, on the premise of introducing the global optimal solution, the adaptive adjustment factors and are introduced, and the search formula of the improved ABC algorithm is obtained:

$X_{x j}^{\text {new }}=W_{1} \times X_{i j}+W_{1} \times r_{1} \times\left(X_{x j}-X_{\text {neighbour }, j}\right)+W_{2} \times r_{2}\left(y_{j}-x_{x j}\right)$

Among them,

$$
\begin{aligned}
& W_{1}=W_{\text {max }}-\left(2-e^{\frac{\text { Iterate }}{\text { max cycle }} \ln 2}\right)\left(W_{\text {max }}-W_{\text {min }}\right) \\
& W_{2}=W_{\text {min }}+\left(2-e^{\frac{\text { Iterate }}{\text { max cycle } I n 2}}\right)\left(W_{\text {max }}-W_{\text {min }}\right)
\end{aligned}
$$

Iterate is the current number of iterations; maxcycle is the maximum number of iterations; $W_{\min }$ is the minimum value of the adjustment factor; $W_{\max }$ is the maximum value of the adjustment factor; $W_{1}$ and $W_{2}$ represent the proximity of the new honey source to the original honey source, the neighboring honey source and the population optimal honey source speed.

\subsection{Improve Following Bee Selection Probability}

In order to prevent the premature convergence of the population and fall into a local minimum, a reverse roulette selection mechanism is introduced, which aims to follow the bee mining is a poorly adapted honey source, avoiding the population to quickly evolve to a highly adaptable honey source Concentration, thus maintaining the fitness of the population, so the 
probability $P_{i}$ formula of the bee colony and solution $X_{i}$ is changed to:

$$
P_{i}=\frac{1 / \text { fit }_{i}}{\sum_{j=i}^{m}\left(1 / f_{i t}\right)}
$$

In the early stage of the algorithm, due to the introduction of the reverse roulette selection mechanism, the following bees will focus on searching for honey sources with poor adaptability, thereby slowing the convergence rate of the algorithm. Therefore, this article introduces an adaptive judgment factor of $\sigma, \sigma=e^{\frac{\text { Iterate }}{\text { maxcycle } I n 2}}-1$.In the early stage of the algorithm, the following bees can be used to search for honey sources with high adaptability to ensure the convergence speed. In the latter part of the algorithm, the following bees can search for honey sources with low adaptability to ensure the diversity of the population and avoid falling into local optimal values. Therefore, the probability $P_{i}$ formula of the bee colony and solution $X_{i}$ is optimized as:

$$
P_{i}= \begin{cases}\frac{\text { fit }_{i}}{\sum_{j=1}^{m} \text { fit }_{j}} & \text {, rand }>\sigma \\ \frac{1 / \text { fit }_{i}}{\sum_{j=1}^{m}\left(1 / \text { fit }_{j}\right)} & \text {, rand }<\sigma\end{cases}
$$

\section{BP Neural Network based on ABC Algorithm}

The process of updating the weights of the BP neural network to the $\mathrm{ABC}$ algorithm can speed up the convergence and avoid falling into a local minimum. The specific steps to improve the artificial bee colony algorithm $(\mathrm{ABC})$ to optimize the $\mathrm{BP}$ neural network include the following parts:

- According to the input samples and output requirements, establish a BP neural network with different meteorological environment. Determine the neural network structure.

- Initialize the BP neural network.

- Initialize the parameters of the $\mathrm{ABC}$ algorithm: the size of the bee colony, the maximum number of iterations, the number of stays, etc.

- Conduct bee picking, follow the operation of bee and detective bee, and find the best honey source.

- Return the best honey source to the BP neural network. The weights and thresholds of the BP neural network are replaced with the calculated optimal solutions. Input a certain prediction day to test the improved BP neural network and output the predicted value.

\section{Simulation Verification}

In the MATLAB simulation experiment, select the experimental parameters: the size of the bee colony is 200 , the number of bees is 100 , the number of bees is 100 , the limit value is 100 , and the maximum number of cycles is 50. The improved ABC-BP neural network model is compared with the traditional BP network model for photovoltaic power prediction. As shown in Figure 1, the improved ABC-BP model predicts that the absolute error between the generated power and the expected generated power is within $20 \%$. As shown in Figure 2, the absolute error between the predicted BP model and the expected generated power exceeds 20 in multiple time periods. \%. It shows that the improved ABC-BP neural network has higher prediction accuracy.

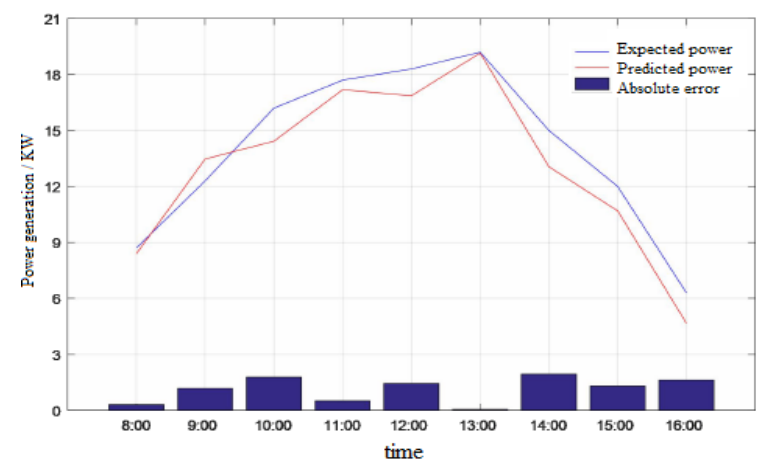

Figure1 ABC-BP predicted power generation

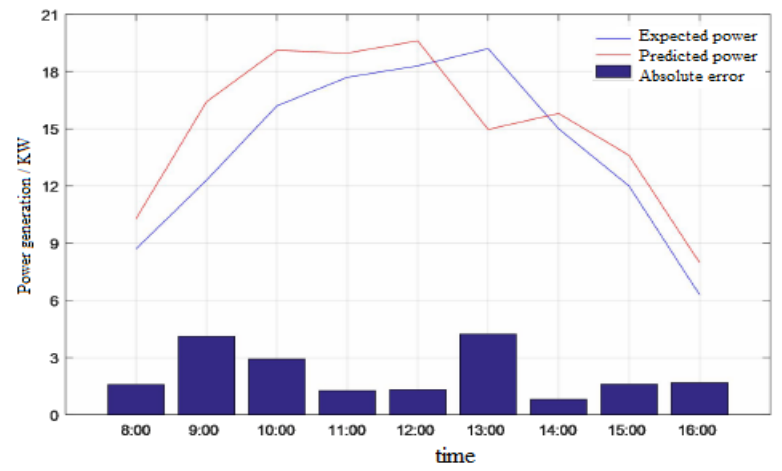

Figure2 BP predicted power generation

It can be seen from Figure 3 that the optimal and worst of the improved ABC-BP neural network is lower than the optimal and worst of the traditional BP method, indicating that the improved has higher accuracy and can be more effective under the same training times Quickly approach the optimal solution. 


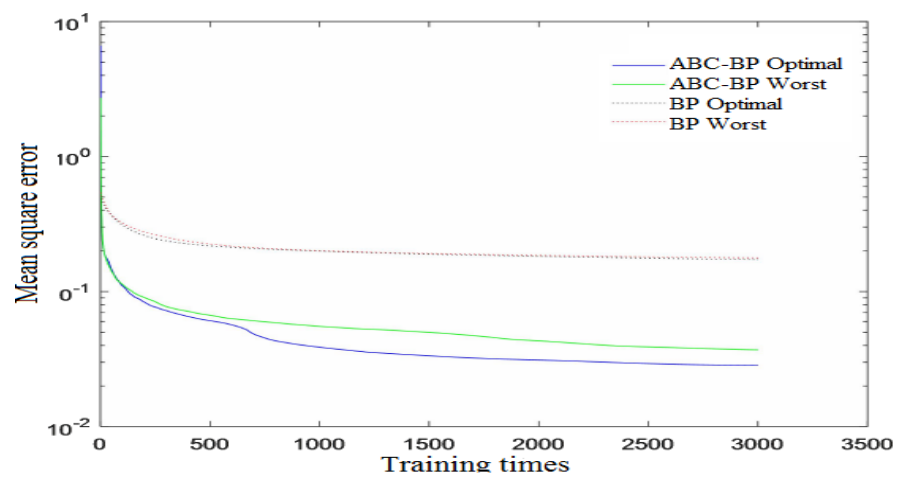

Figure3 the mean square error curve of power prediction
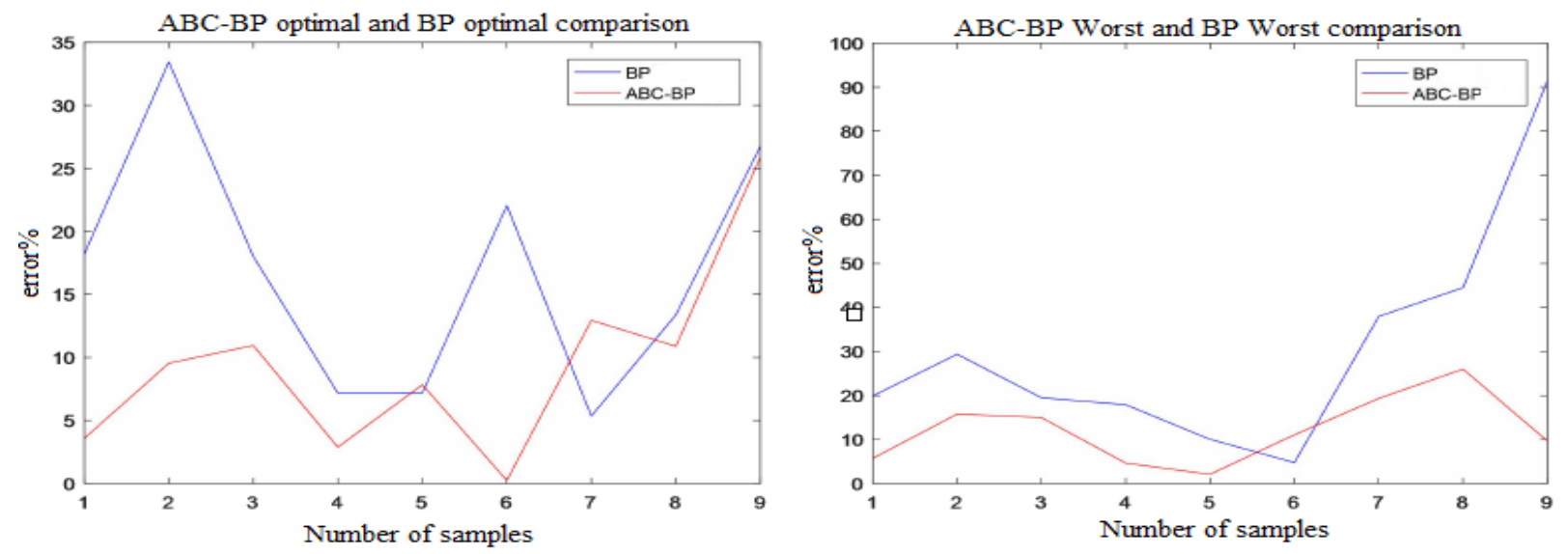

Figure 4 Comparison of training error between BP network and ABC-BP network

It can be seen from Figure 4 that the improved artificial bee colony algorithm is used to optimize the BP network, and the process of searching the optimal weight and threshold value of the neural network is converted into the process of searching for the best honey source by the ABC algorithm. ABC-BP optimized neural network prediction model has higher accuracy and convergence speed, which can effectively predict the change of generated power and provide a more accurate reference for power dispatch.

\section{Conclusion}

BP neural network has the shortcomings of slow convergence and easy to fall into local minimum. In this paper, the improved artificial bee colony algorithm is used to predict the photovoltaic power generation in the neural network. The artificial bee colony algorithm is improved and applied to the BP neural network. Under the weather conditions, the time correlation between similar daily power generation data, the optimal solution is optimized, and the convergence speed and accuracy of the neural network are improved.

\section{Acknowledgements}

School level project: Research on performance optimization of double-sided module of FuKang photovoltaic power station No: 801/204190464

\section{References}

1. Mao Jian, Zhao Hongdong, Yao Jingjing. The development and application of the artificial and artificial Jingjing network [J]. Electronic Design Engineering, 2011, 19 (24): 62-65

2. Ding Ming, Wang Lei, Bi Rui. Short-term prediction model of output power of photovoltaic power generation system based on improved BP neural network [J]. Power System Protection and Control, 2012,40 (11): 93-99+ 148 .

3. Li Pengmei, Zang Chuanzhi, Wang Kankan. Forecast of photovoltaic power generation based on similar day and neural network [J]. Renewable Energy,2013, 31 (10): 1-4 + 9.

4. Zhong Jianwei, Liu Junfu, Zhou Wenhui, etc. A BP Neural Network Optimization Algorithm in Distribution Network Fault Location [J] .Journal of Inner Mongolia Normal University (Natural Science Chinese Edition), 2017, 46 (4): 498-501.

5. Li Long, Wei Jing, Li Canbing, et al. Load model prediction based on artificial neural network [J].Journal of Electrical Engineering and Technology, 2015,30 (08): 225-230.

6. KARABOGA D, OZTURK C. Neural networks training by artificial bee colony algorithm on pattern classification [J]. Neural Network World,2009, 19 (3): 279-292. 
7. KARABOGA D, BASTURK B. A powerful and efficient algorithm for numerical function optimization: artificial bee colony ( $\mathrm{ABC}$ )algorithm [J]. Journal of Global Optimization, 2007, 39 (3): 459-471.

8. Hassim Y M M, Ghazali R.Optimizing functional linkneural network learning using modified bee colony on multi-class classifications[C]//Lecture Notes in Electri-cal Engineering 279: Advances in Computer Science and its Applications, 2014: 153-159. 